Conference

\title{
Effects of the binder material on the mechanical properties of thick-film magnetostrictive materials
}

\author{
N.J. Grabham*, S.P. Beeby, N.M. White \\ School of Electronics and Computer Science, University of Southampton, Highfield, Southampton SO17 1BJ, UK \\ Received 4 August 2003; received in revised form 4 August 2003; accepted 4 September 2003
}

\section{Abstract}

This paper presents research carried out at the University of Southampton into the development of a magnetostrictive thick-film material suitable for use with silicon micromachined devices. This form of magnetostrictive material has previously been deposited onto alumina substrates and this paper reports further work on migrating the technology onto silicon. The evaluation of two alternative glass frits for use as the binder within the thick film is reported. The correct choice of the binder material is important in a thick-film material because it is responsible for binding the active material within the thick film into a composite material and also adhering the film to the substrate. A series of tests have been applied to samples fabricated using various glass frits to assess their mechanical properties and suitability for the micro-actuator applications.

(c) 2003 Published by Elsevier B.V.

Keywords: Thick film; Magnetostrictive; MEMS

\section{Introduction}

Magnetostrictive materials exhibit a dimensional change under the influence of a magnetic field. As the actuation is achieved in a non-contact manner with this material, it is not necessary to provide physical connections to the sample, as required with, say, piezoelectric materials. The potential for wire-free operation is advantageous in the field of micro-electro-mechanical system (MEMS) devices, some of which have been fabricated using thin-film techniques to deposit a range of magnetostrictive materials. An example of such a device is the micropump described by Quandt and Seemann [1].

One of the disadvantages of thin-film deposition is the relatively low deposition rate, which tends to produce films typically less than $1 \mu \mathrm{m}$ in thickness. Thick-film technology is capable of producing films up to several $100 \mu \mathrm{m}$ thick. All film technologies, however, result in the production of layers having different physiochemical properties from that of the bulk material.

The thick-film fabrication technique used to prepare the magnetostrictive materials described in this paper provides

* Corresponding author. Tel.: +44-23-8059-5162; fax: +44-23-8059-2901.

E-mail address: njg@ecs.soton.ac.uk (N.J. Grabham). a low-cost method of producing films with thicknesses in excess of $100 \mu \mathrm{m}$. The process is based upon a screen printing technique that is believed to have been in use for over a thousand years as a form of graphic art reproduction. The processing equipment used in this study is designed for use in the fabrication of electronic circuits and sensors. With thick-film technology, the target film material is mixed with a binder material, often a devitrifying glass frit, and suitable solvents to produce a printable paste. This paste is then screen printed through a patterned screen onto a suitable substrate, typically alumina, insulated steel or silicon. The printed paste is then allowed to settle prior to drying, which is necessary to remove the solvents within the composition. The final stage of the fabrication process is the firing stage. During this phase, the materials within the film are exposed to temperatures up to $850^{\circ} \mathrm{C}$ in a continuous belt furnace.

The result is a fired composite thick-film material.

The magnetostrictive thick-film pastes used in this work contain the giant magnetostrictive material Terfenol-D, in a powdered form, as the active material. Two alternate glass frits are evaluated for use as the binder material within the thick film. To improve the structure of the fabricated thick film, an inert filler material is added to the paste to reduce voids. The active material is the same as that used in the fabrication of epoxy bonded magnetostrictive composites, such as those described by Duenas et al. [2]. 


\section{Fabrication}

To produce the magnetostrictive pastes used in this work, the following quantities of dry materials were used: $15 \mathrm{~g}$ of sieved Terfenol-D powder (particle size less than $160 \mu \mathrm{m}$ ); $6 \mathrm{~g}$ of alumina powder; and $6.75 \mathrm{~g}$ of glass frit binder. Sieved Terfenol-D powder is used because the material supplied by the manufacturer includes particles that are too large for compatibility with the screens used in the printing process. Use of over-size particles in the paste causes clogging of the screen, which results in incomplete prints.

Initially the appropriate dry weights of the alumina filler material and glass frit were mixed together using a pestle and mortar. During this mixing process, powder agglomerates will be broken up. To these mixed powders, sufficient vehicle was added to produce a paste, which was then processed using a triple-roll mill to further mix the two powders, ensuring an even distribution of glass frit and alumina through the paste. Once this paste has been removed from the triple-roll mill, the corresponding quantity of sieved Terfenol-D powder was added. The paste was then mixed using a spatula, and additional vehicle added until a suitable paste viscosity was reached. Once sufficient vehicle had been added the paste was again mixed in the triple-roll mill to improve the distribution of the various components. After this stage the paste was transferred to a glass container and left to stand. After a short while it returned to its original (pre-milling) viscosity and was then ready for use.

The printing and firing of the thick films are achieved using the same techniques as those used for printing magnetostrictive thick films onto alumina substrates as described by Grabham et al. [3]. In this current work, the alumina substrates have been replaced with $100 \mathrm{~mm}$ diameter, $530 \mu \mathrm{m}$ thick, microelectronic grade silicon wafers. These have a $1 \mu \mathrm{m}$ thick layer of thermally-grown silicon dioxide on their surface to improve the chemical bonding between the thick film and the silicon substrate.

After the firing process, the fabrication of the thick films is complete and the samples are ready for separating into individual devices. This is achieved by using a standard diamond wafer saw. This yields individual test samples that are ready for the measuring process. After sawing, the substrates have dimensions of $12.8 \mathrm{~mm}$ wide by $88 \mathrm{~mm}$ long. The thick-film region is $11 \mathrm{~mm}$ wide by $45 \mathrm{~mm}$ long centred in the width of the substrate and with the end of the thick film situated $32 \mathrm{~mm}$ from the free-end of the beam. The thick films have a nominal thickness of $150 \mu \mathrm{m}$ after firing.

Two different glass frits were evaluated for use as the binder material in this work. The glass frits used were Corning CF7575, a lead-zinc-borosilicate devitrifying glass; and Ferro EG2760, a thick-film passivation material. The batches of magnetostrictive pastes prepared for these experiments were of the same composition differing only by the choice of glass frit. The fabrication process was also kept consistent between batches on order to ensure that any behavioural changes were only due to the binding matrix.

\section{Measurement techniques}

To assess the effect of the binder material on the mechanical properties of the magnetostrictive thick films, a number of tests were carried out. These are described below.

\subsection{Tape and scratch tests}

These are standard tests that are widely used within the hybrid microcircuit industry. In the tape test, a strip of adhesive tape is placed across the surface of the fired thick film, left for several seconds and then removed. Any material on the surface of the thick film that is poorly bonded will come away with the tape and is clearly evident from observation by eye. In the scratch test, a metal stylus is used to scratch the thick film off the substrate. This tests the adhesion between the thick film and the substrate. The amount of force required to mark the surface of the thick film can be used as an indication of its strength.

\subsection{Visual inspection}

The surfaces of the fired thick films and, after cleaving, their cross-sections were inspected using a scanning electron microscope (SEM) to enable the structure of the fired films to be examined. This allows the distribution of the active and filler materials to be observed and the presence of any inclusions to be identified.

\subsection{Young's modulus}

The Young's moduli of the thick films were determined by loading tests and finite element analysis (FEA). The FEA approach was used so that the anisotropic nature of the silicon substrate can be taken into account. The use of computational modelling also allows samples with non-trivial geometry to be readily studied.

The loading tests require the sample to be supported in a cantilever beam configuration, which is then loaded by adding masses to the free-end. The deflection of the beam is recorded for increasing load. The results are then used to calculate a deflection for a given arbitrary loading. This is then input into the FEA model and the Young's modulus of the thick film adjusted until the computed deflection matches that corresponding to the chosen arbitrary loading.

\subsection{Frequency response}

The frequency response of the magnetostrictive samples was recorded and this can be used, in conjunction with FEA modelling, to determine the net magnetostriction of the film under test. The frequency response is achieved by actuating a beam containing the film within a magnetic field and measuring the displacement of the free-end of a cantilever beam. An optical interferometer, based on the design of one used to measure micromachined devices [4], is used to record the 
deflection. This can be used to compare the performance of different films, subject to them having similar dimensions and substrate material. The deflection can also be used with FEA modelling to enable a value for the net magnetostriction of the thick film to be determined.

\section{Results}

\subsection{Tape and scratch tests}

The scratch and tape tests were performed on samples of both thick films. For the film containing the CF7575 glass frit, a small amount of surface material was removed during the tape test. Repetition of the tape test caused no further significant removal of material from the same area. This is indicative of the presence of loose material on the surface of the thick film, with the main bulk of the layer being adequately bonded. In the scratch test, a moderate force was required to mark the thick film using a metal stylus.

In the case of the sample fabricated using the EG2760 glass frit, less material was removed during the tape test than was observed for the CF7575 based film. This suggests that the bonding of the material within the EG2760 based film is superior to that of the CF7575 based film. Again, the scratch test required a moderate force to mark the thick film so no comparisons between the two glass frits can be drawn from the scratch tests.

\subsection{Visual inspection}

The surfaces and cross-sections of the thick films were examined using a SEM and both types of film were found to exhibit similar structure. This indicates that the choice of binder material does not affect the distribution of the active material and filler particles within the fired film. A SEM image of a typical thick-film surface is shown in Fig. 1. The larger, angular, Terfenol-D particles can be seen to be surrounded by the smaller alumina particles, which serve to fill in voids within the thick film that are present because of the irregular shape of the larger Terfenol-D particles.

\subsection{Young's modulus}

The Young's modulus was determined for samples of both film types using the loading technique outlined previously. The CF7575 based thick film was found to possess a modulus of $33.5 \mathrm{GPa}$, whilst the film containing EG2760 glass frit exhibited a lower modulus of $22 \mathrm{GPa}$. The higher modulus of the CF7575 based film may suggest that it is more rigidly bonded as the resulting material is stiffer. The actual modul of the glass frits, however, is not known, so the effect of this on the overall moduli of the structure cannot be quantified.

\subsection{Frequency response}

The frequency response of the samples was observed for frequencies around the mechanical resonance of the sam-

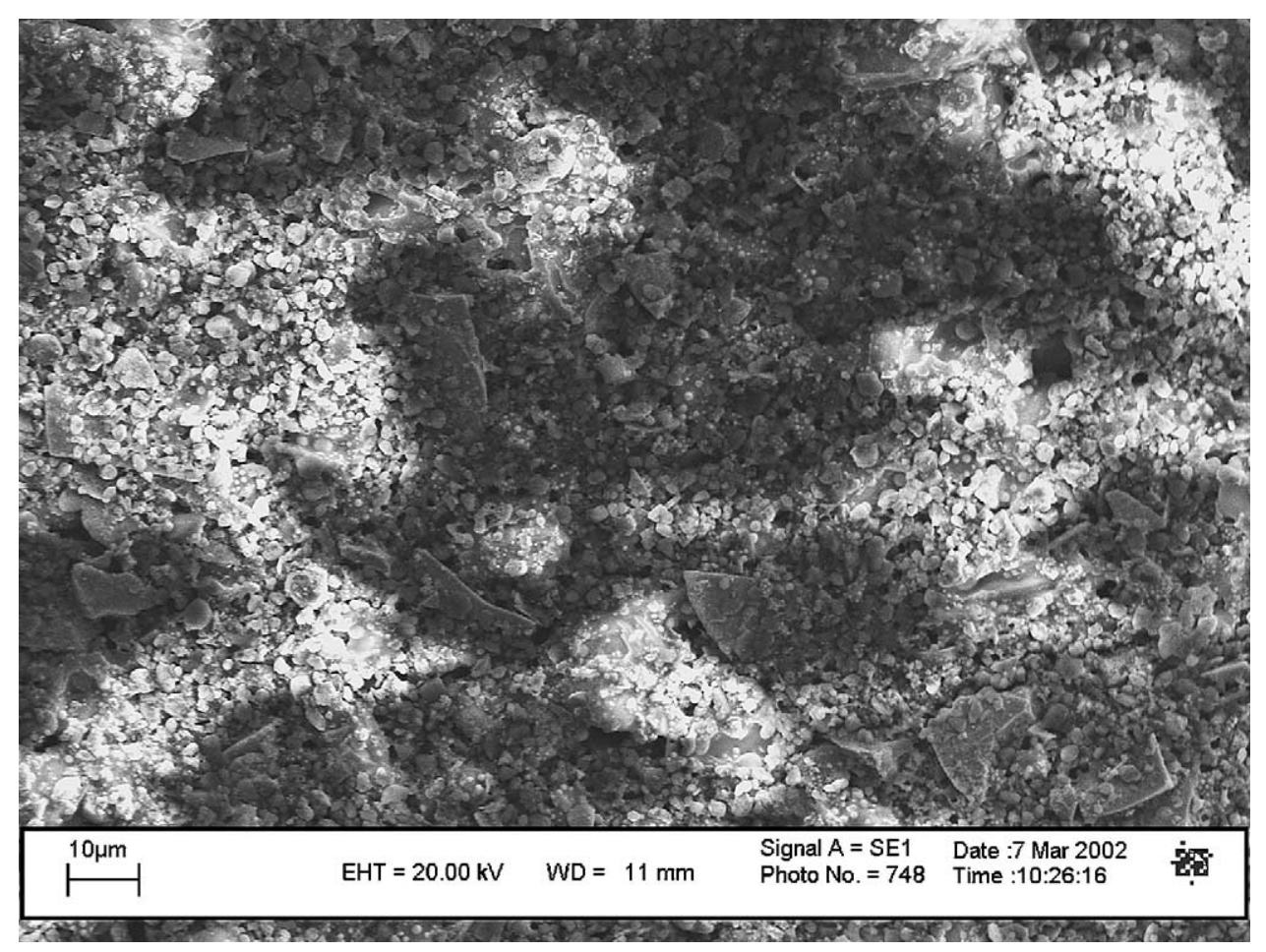

Fig. 1. Typical surface of thick film. 


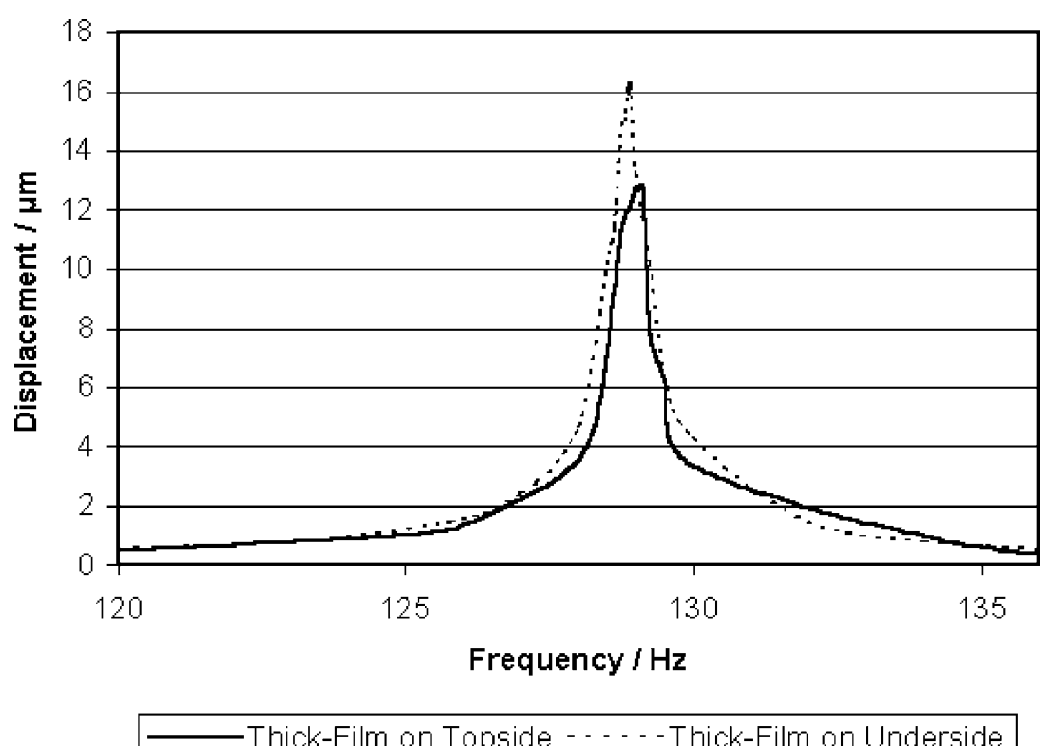

Fig. 2. Effect of changing from tensile to compressive loading on thick film containing CF7575 glass frit.

ple (approximately $110 \mathrm{~Hz}$ for an unloaded sample), when mounted in a cantilever configuration as described earlier. The excitation magnetic field was kept at the same strength for all tests and was measured to be $5.19 \mathrm{kA} / \mathrm{m}$. The frequency response measurements were performed for both types of thick film under various pre-loading conditions, and the results then examined.

The loading conditions investigated included self-mass loading, where the loading is solely provided by the mass of the sample itself (mainly the beam), in both compressive and tensile configurations. These were achieved by positioning the sample with its plain orientated horizontally, and the thick film on either the upper or lower face of the substrate. This introduces tensile loading when the thick film is on the upper side, and compressive loading when it is on the lower face.

The other loading condition examined involved investigating the effects of increasing the compressive loading through the addition of mass to the free-end of the sample. This additional mass was formed using small squares of alumina, which would not interfere with the magnetic field around the sample.

The effect of using both tensile and compressive self-mass loading was investigated initially. It was found that the application of compressive loading had the effect of increasing the observed deflection from that recorded for the same sample under tensile loading. A comparison of the difference between these two loading conditions can be seen in the graph shown as Fig. 2, which shows the frequency responses obtained for both tensile and compressive loading of the same sample on common axis. Based upon these findings, the effects of further increasing the compressive loading were investigated for both types of thick film.

\subsection{Effects of increasing compressive loading}

To investigate the effects of increasing the compressive loading of the thick film, frequency responses were recorded for each of the samples with the loading varied between zero additional mass, in which case the sample's own mass pro-

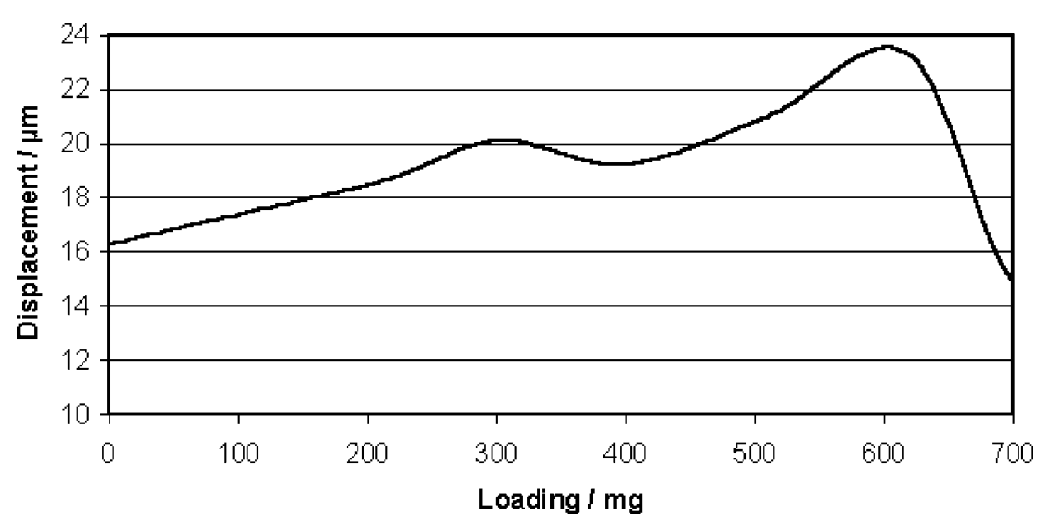

Fig. 3. Effect of compressive loading on thick film containing CF7575 glass frit. 


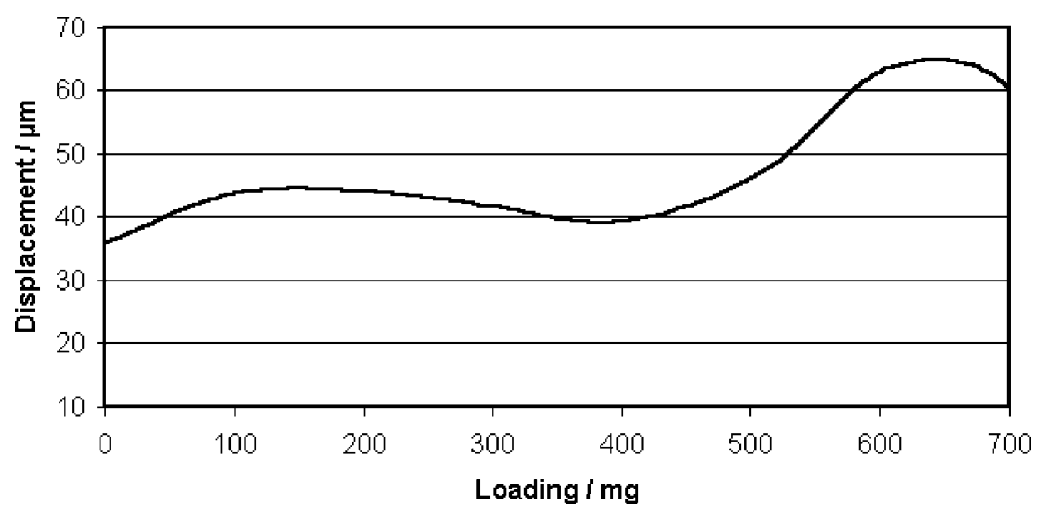

Fig. 4. Effect of compressive loading on thick film containing EG2760 glass frit.

duces compressive loading, and $700 \mathrm{mg}$ of additional mass. The loading mass was increased in $100 \mathrm{mg}$ steps.

The recorded dynamic displacements of the free-end of the beam for the thick films based upon CF7575 and EG2760 glass frits can be seen in Figs. 3 and 4, respectively. In both cases, it can be seen that the application of additional loading causes an increase in the deflection observed at the structure's resonance. This increase is present in both cases until a loading of approximately $600 \mathrm{mg}$ is reached. For loading in excess of this value, the observed deflection was seen to reduce for both types of thick film under test. As these measurements were made at the resonant frequency of the test samples, it is necessary to take the $Q$-factor of the structure into account and compute an equivalent static deflection. The $Q$-factor is obtained from the individual responses for each of the loading conditions. The resulting equivalent static deflections, normalised to the deflection at zero additional loading, are plotted in Fig. 5 for the thick film containing CF7575 glass frit, and Fig. 6 for that containing the EG2760 glass frit. The results from the thick film

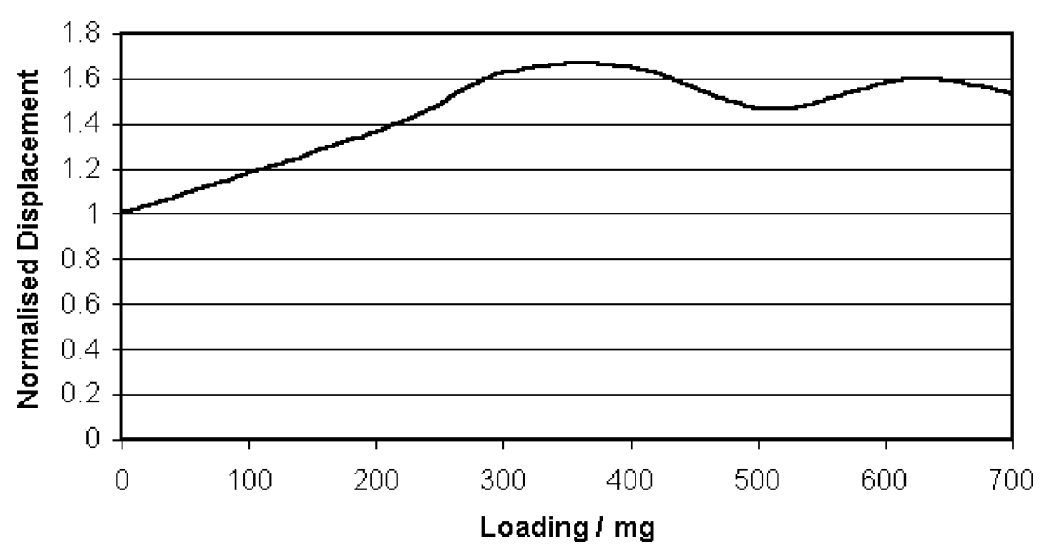

Fig. 5. Normalised static deflection of thick film containing CF7575 glass frit.

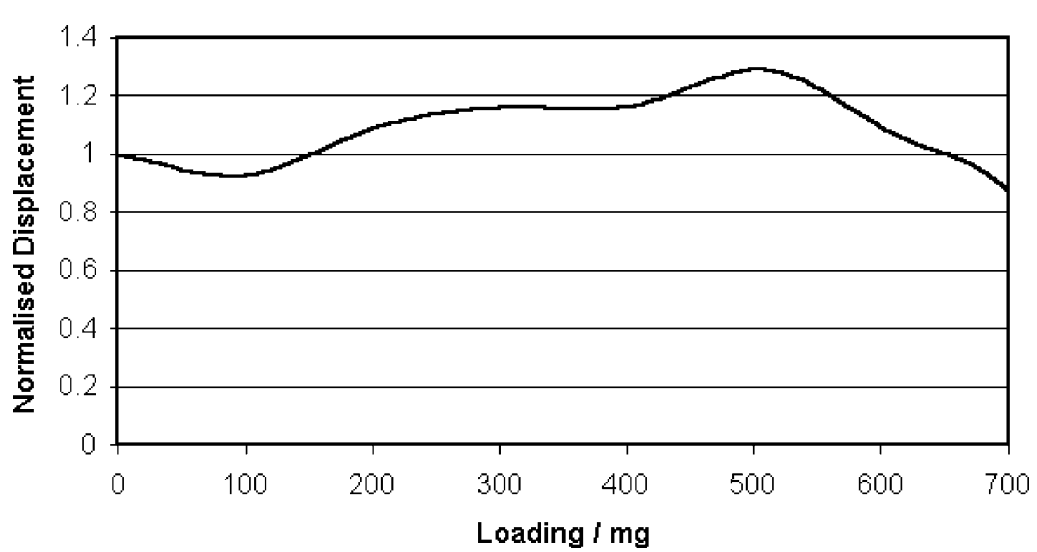

Fig. 6. Normalised static deflection of thick film containing EG2760 glass frit. 
containing the CF7575 glass frit shown that for additional loading in the range $300-400 \mathrm{mg}$ the equivalent static deflection is increased by approximately $60 \%$ from that observed for zero additional loading. For loading over $400 \mathrm{mg}$ the increase in static deflection starts to reduce. Such a marked improvement is not shown by the thick film incorporating the EG2760 glass frit as the maximum increase is of the order of $30 \%$, which is achieved with a loading of $500 \mathrm{mg}$ after which the deflection starts to reduce.

\section{Discussion}

5.1. Advantage of compressive loading over tensile loading

The effect of changing from tensile to compressive load, which was shown in Fig. 2, is to increase the observed deflection. It is proposed that this is attributable to the compressive loading providing a pre-load (or pre-stress) to the thick film, thereby causing the particles within the film to be in closer contact. This in turn means that when the active Terfenol-D particles undergo a dimensional change, upon the application of the actuating magnetic field, the strains produced by the particles are better coupled into the composite as a whole. This causes an increase in the net magnetostriction of the thick film, and hence an increase in the observed deflection.

\subsection{Results of increased compressive loading}

The effect of increasing the compressive loading, up to a point, has been seen to further increase displacements recorded for both film types, both in the dynamic values and also in the computed equivalent static results. These increases are attributed again to a closing up of the structure within the thick film, leading to improved coupling of the produced strains into the composite as an whole. As the loading continues to increase, a point is reached after which the benefits of pre-stressing the thick film are outweighed by the increased loading that the material has to work against, and therefore the resulting deflection starts to reduce.

\section{Conclusions}

This paper has presented results of a study into the suitability of two glass frits, Corning CF7575 and Ferro EG2760, for use as the binder material in a magnetostrictive thick-film material that is suitable for deposition onto silicon substrates pre-coated with a $1 \mu \mathrm{m}$ thick interface layer of silicon dioxide. Of the two glass frits tested the Ferro EG2760 was found to produce a thick film, that when deposited onto silicon substrates and excited using a magnetic field of $5.19 \mathrm{kA} / \mathrm{m}$, exhibited greater actuation than comparable thick films containing the Corning CF7575 glass frit as the binder material. It has also been seen that the appli- cation of a compressive pre-stress to the magnetostrictive thick film can be used to increase the observed deflection, and thus the net magnetostriction of the thick film. In the case of the thick film containing the Ferro EG2760 glass frit as the binder, a loading of $500 \mathrm{mg}$ was found to give the largest increase in the calculated equivalent static deflection.

\section{Acknowledgements}

The authors wish to thank the Engineering and Physical Sciences Research Council (EPSRC) for their financial support under grant number GR/R43327. We also gratefully acknowledge the support and assistance given to us by Newlands Technology Ltd.

\section{References}

[1] E. Quandt, K. Seemann, Magnetostrictive thin film microflow devices, Proc. Micro Syst. Technol. 96 (1996) 451-456

[2] T.A. Duenas, L. Hsu, G.P. Carman, Magnetostrictive composite material systems analytical/experimental, Mater. Res. Soc. Symp. Proc. 459 (1997) 527-543.

[3] N.J. Grabham, S.P. Beeby, N.M. White, The formulation and processing of a thick-film magnetostrictive material, Meas. Sci. Technol. 13 (2002) 59-64.

[4] M.V. Andres, K.H.W. Foulds, M.J. Tudor, Analysis of an interferometric optical fibre detection technique applied to silicon vibrating

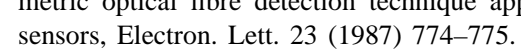

\section{Biographies}

Neil J. Grabham received the degree of MEng in information engineering from the University of Southampton in 1998 and was awarded his $\mathrm{PhD}$ in 2002. He is currently a research fellow in the School of Electronics and Computer Science and is working on the development of thick-film magnetostrictive material for actuator applications in MEMS devices. His interests include thick-film materials and the application of microprocessors to sensor applications.

Steve P. Beeby obtained his BEng (Hons) in mechanical engineering in 1992 and was awarded his PhD in 1998. He is currently a research fellow in the School of Electronics and Computer Science and is researching in the field of micro-electro-mechanical systems (MEMS). In particular, his research involves the development of fabrication processes whereby thick-film piezoelectric materials can be combined with micromachined silicon structures. His skills include the finite element modelling and design of MEMS devices, silicon processing and MEMS packaging, and testing. He currently has over 40 publications in the field.

Neil M. White is professor of intelligent sensor systems within the Schoo of Electronics and Computer Science at the University of Southampton and also director of the Institute of Transdicer Technology. He was awa PhD in 1988 for a thesis on the aplicato of thick-film piezanded a for load cells. Professor While was apoined as lecturer in 1990, senior lecturer in 1999, reader in 2000 and currently holds a personal chair. He has published extensively in the area of thick-film sensors and intelligen instrumentation and is author or co-author of over one hundred scientific publications. He is a fellow of the Institute of Physics, a chartered engineer and a senior member of the IEEE and has served on several committees in various professional bodies. 